\title{
ANALYSIS OF MOBILE APPLICATIONS FOR DIABETES SELF-MANAGEMENT IN THAILAND
}

\author{
Maneerat Rattanamahattana and Palung Rungsa \\ Faculty of Pharmaceutical Sciences, Khon Kaen University, Khon Kaen, Thailand
}

\begin{abstract}
With advances in mobile technology, use of mobile devices like smartphones, has grown rapidly around the world including Thailand. Many mobile applications (apps) have been launched to help patients with chronic non-communicable diseases to manage their health behavior. However, little is known about the apps available in commercial application stores. Our study aimed to explore and evaluate the content and features of free download mobile apps available for diabetes self-management according to eight categories: (1) information support; (2) subscription requirement; (3) notifications; (4) visual aids; (5) goal setting; (6) export data; (7) medical consultation; and (8) compatibility. We found 22 apps available in the AppStore and 84 apps in the Google Play store. The applications available in the App Store could be categorized as tracking only (23\%) as well as tracking and education (77\%) with the three most common features; visual aids $(95 \%)$, export data (82\%), and notification (73\%). The applications on Google Play store could be categorized into 4 groups; tracking (35\%), tracking and education (15\%), education alone (29\%), and diabetes food recipes $(21 \%)$. The apps in the tracking and tracking and education groups had a wide range of features, with app scores ranging from one to seven. Apps in the education and diabetes food recipe groups only had one feature - information support. Many apps also contained advertisements, with no references provided. Therefore, it may be practical that healthcare providers explore each application before recommending to their patients. Mobile apps that specifically fit the Thai setting, with quality assessment of the reliability of information as well as assessment of e-health literacy are needed for diabetes self-management among Thai patients.
\end{abstract}

\section{KEYWORDS}

Diabetes Mellitus, Self-Management, Mobile Devices, m-Health, Thai

\section{INTRODUCTION}

Diabetes mellitus is a non-communicable disease (NCD), of which an alarming increase of diabetes patients has been reported of with over 1.6 million deaths worldwide in 2016 (WHO, 2018) and it is the third leading cause of all deaths among NCDs in Thailand (Ministry of Public Health Thailand, 2016). Previous studies suggest that use of mobile phone apps in diabetes management can modestly improve glycemic control (Wayne and Ritvo, 2014; Kirwan, Vandelanotte, Fenning \& Duncan, 2013). In fact, smartphones have gained rapidly popular use across the population. The proportion of people who possess smartphones in Thailand was $82.1 \%$ and the trend of use among baby boomers aged 51-69 has been growing significantly. The aim of this study was to explore and evaluate contents and features of diabetes self-management applications available in Thailand.

\section{METHODS}

This was a descriptive study using online search for diabetes-related applications available in Thailand from two popular platforms i.e. Android Google Play Store and iOS AppStore by using two mobile devices - Sony Xperia XA Ultra and iPhone SE, respectively. The key terms for diabetes self-management applications included both in English ('diabetes', 'diabetes management') and in Thai ('เบาหวาน'). The inclusion criteria included the applications that were specifically designed for patients regarding diabetes self-management with free download; provided in either English or Thai language; used with smartphones; and updated on a 
regular basis; and with no duplicates. Descriptive information regarding the name of developer, latest update, and operating system of the applications was collected. Based on the exhaustive literature review (Izahar et al, 2017; Demidowich, Lu Tamler, \& Bloomgarden, 2012), the applications were then categorized and evaluated the availability of the following features; (1) information support; (2) subscription requirement; (3): notifications; (4) visual aids; (5) goal setting; (6) export data; (7) medical consultation; and (8) compatibility. A point would be given for each available feature for the total score across all applications included in the study.

\section{RESULTS}

From the above inclusion and exclusion criteria, 22 apps on AppStore and 84 apps on Android Google Play Store were selected. The content found in AppStore apps could be classified in two categories i.e. tracking blood sugar of patients (77\%) and the combination of tracking and patient education (23\%). The apps from Google Play store contained more varieties as shown in Figure 1. The distribution of features in the apps from both iOS and Android is compared in The most popular features for iOS were visual aids (95\%) followed by export data (82\%), notification (73\%), compatibility (50\%), goal setting (41\%), subscription (32\%), information (23\%), and medical consultation was the least popular feature provided in only $9 \%$ of the total 22 iOS apps. Information feature was the most popular feature in Android apps (65\%) followed by visual aids (46\%), export data (38\%), notification (33\%), compatibility (22\%), goal setting (19\%), subscription (8\%). Similarly to the iOS apps, medical consultation was the least popular feature available (5\%) for Android. Considering the distribution of the features available for each Android app, there was a wide range of scores from 1 to 7 features (Figure 2). Most of the apps available on AppStore contained more than 4 features while the apps from Google Play Store contained less than 4 features. However, none of the apps from either platforms obtained a full score of 8 . In addition, the apps that provided information in Thai language were still very rare.

Recent evidence suggests the use of mobile technology as a tool for self-management has the potential to fulfill the needs of patients, healthcare providers, or both (Goyal and Cafazzo, 2013). In this study, both the Android and iOS platform provided diabetes self-management apps tended to provide tracking and education, but the Android apps had a wider variety of categories than the iOS apps. For instance, Android apps provided more education features and suggested diabetes recipes. However, across all of the apps, most of the content was provided as information without any evidence-based citations or references. Thus, there is no guidance regarding the reliability of the information. Therefore, lay patients will require high health literacy skills to evaluate the information before they can confidently apply the healthy tips recommended by the apps.

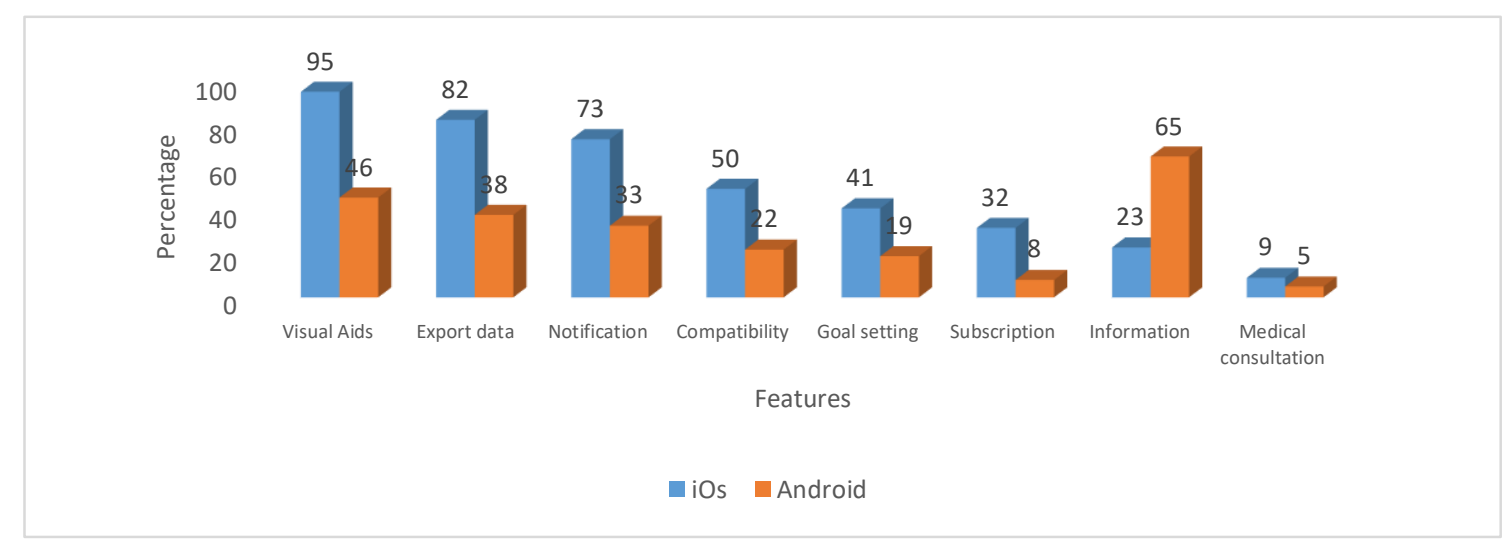

Figure 1. Comparison of available features found on iOS apps versus Android apps 


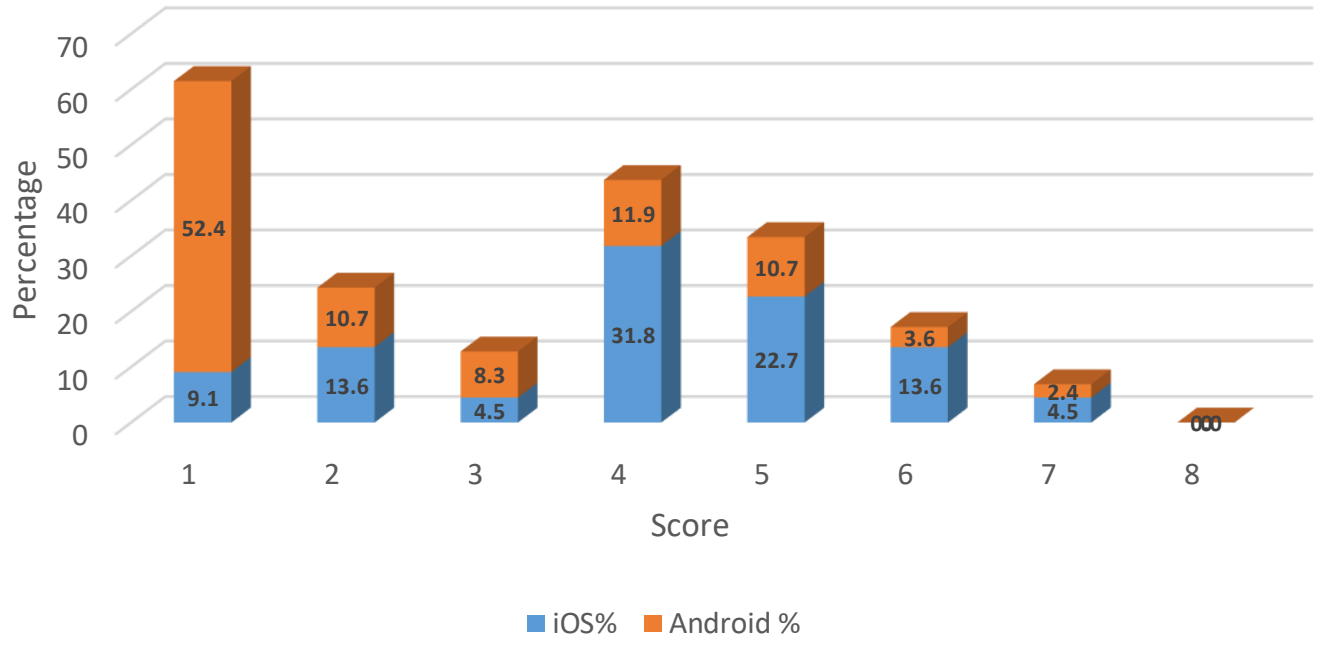

Figure 2. Proportion of score regarding features on iOS apps versus Android apps

\section{CONCLUSION}

The contents and features of free download mobile apps available for diabetes self-management in both iOS and Android are plethora. However, mobile apps that specifically fit the Thai setting i.e. language and lifestyle should be further developed. Moreover, the apps should be assessed for the quality and reliability of the information they provide and investment in e-health literacy is needed for the effective use of these apps in the self-management of diabetes among Thai patients.

\section{REFERENCES}

Demidowich AP, Lu K, Tamler R, Bloomgarden Z., 2012. An evaluation of diabetes self-management applications for Android smartphones. J Telemed Telecare 18(4):235-8.

Izahar, S., Lean, Q. Y., Hameed, M. A., Murugiah, M. K., Patel, R. P., Al-Worafi, Y. M., Wong, T. W., ... Ming, L. C., 2017. Content Analysis of Mobile Health Applications on Diabetes Mellitus. Frontiers in endocrinology, 8, 318.

Kirwan M, Vandelanotte C, Fenning A, Duncan MJ., 2013. Diabetes self-management smartphone application for adults with type 1 diabetes: randomized controlled trial. J Med Internet Res.;15(11): e235.

Thailand Digital Economy and Society Development Plan, Ministry of Information and Communication Technology:2016:12.

Wayne N, Ritvo P., 2014. Smartphone-enabled health coach intervention for people with diabetes from a modest socioeconomic strata community: single-arm longitudinal feasibility study. J Med Internet Res.;16(6): e149.

World Health Organization, 2018. Noncommunicable diseases country profiles 2018. Geneva: Licence: CC BY-NC-SA 3.0 IGO. 